\title{
Review Article \\ Circular RNAs in Gastric Cancer: Potential Biomarkers and Therapeutic Targets
}

\author{
Jiafeng Ouyang $(\mathbb{D}$, Zhi Long, and Guoqing $\mathrm{Li}$ i \\ Department of Gastroenterology, The Second Affiliated Hospital of the University of South China, Hengyang, \\ 421001 Hunan Province, China \\ Correspondence should be addressed to Guoqing Li; ligq1970@163.com
}

Received 6 February 2020; Revised 29 May 2020; Accepted 1 June 2020; Published 30 June 2020

Academic Editor: Peyman Björklund

Copyright (C) 2020 Jiafeng Ouyang et al. This is an open access article distributed under the Creative Commons Attribution License, which permits unrestricted use, distribution, and reproduction in any medium, provided the original work is properly cited.

Circular RNAs (circRNAs), as a recently established group of endogenous noncoding RNAs, have been involved in the occurrence and development of different malignancies. Gastric cancer (GC) remains a globally significant contributor to death in cancer patients due to insufficient early diagnosis, limited treatment measures, and poor prognosis. An increasing number of studies have found that many circRNAs are dysregulated in GC and are closely associated with its tumorigenesis and metastasis. Thus, circRNAs have the potential to serve as diagnostic and prognostic biomarkers and even therapeutic targets. This review comprehensively summarizes the most recent findings on how circRNAs influence GC progression and their clinical value. In addition, we present several methological deficiencies in the studies and provide some promising ideas for future research.

\section{Introduction}

In the 21 st century, cancer has become one of the leading global health problems with increasing awareness among medical institutions and the general public. According to World Health Organization statistics, in 2018, there were 18.1 million new cases and 9.6 million cancer-related deaths worldwide, with the worst statistics reported for Asia with a cancer incidence and death rates of $48.4 \%$ and $57.3 \%$, respectively [1]. Gastric cancer (GC) is the fifth most common and third leading cause of cancer-related death among all malignancies worldwide, with 1,033,701 new cases and 782,685 deaths reported in 2018 [1]. Meanwhile, the highest incidence of cancer (32.1 per 100,000 men; 13.2 per 100,000 women) and cancer-related death (15.9 per 100,000 persons) was observed in Eastern Asia [2]. The contribution of China to the global cancer burden is significant, especially for the four leading types of cancer (lung, liver, stomach, and esophageal cancers). Indeed, $50 \%$ of GC patients are from China and their prognosis is quite poor, with 5-year overall survival (OS) rates lower than $35 \%$ in $2013-2015$ [3]. GC is the second and fifth most diagnosed malignancy in Chinese males and females, respectively, and one of the main causes of death in cancer patients $[3,4]$. Moreover, as a major cause of GC, the higher prevalence of Helicobacter pylori in China (56\%) compared to those of the United Kingdom (35.5\%) and United States (35.6\%) may be responsible for the high incidence of GC in China [5].

GC can be divided into early and advanced groups according to the depth of cancer invasion. Early GC can be cured by endoscopic or laparoscopic therapy as the lesion is limited to the gastric mucosa and submucosa and there is little lymph node involvement. Advanced lesions, however, often need extensive surgery, which requires distal gastrectomy and additional lymphadenectomy even with advanced systemic therapy $[6,7]$. The prognosis is also significantly different; the 5-year survival rate of early GC patients $(77.7 \%)$ is 6.5 -fold higher than that of metastatic cancer patients (9.0\%); and most advanced-stage patients survive less than 1 year $[8,9]$. However, more than $80 \%$ of Chinese patients are diagnosed with advanced-stage disease due to the lack of specific symptoms [10]. Therefore, there is an urgent need to identify a better method for GC screening and early detection. 


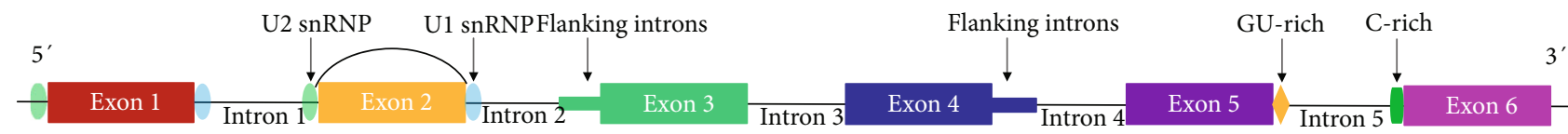

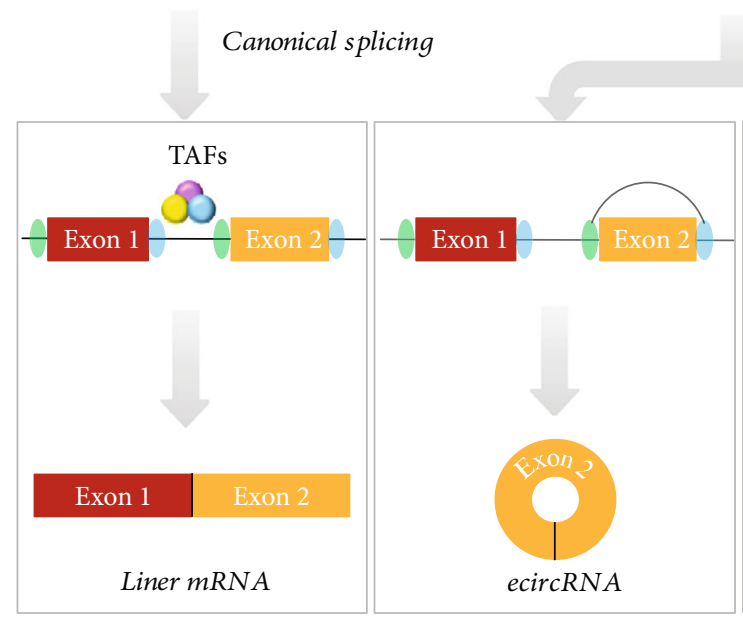

(a)

(b)

Exon skipping

Intron pairing

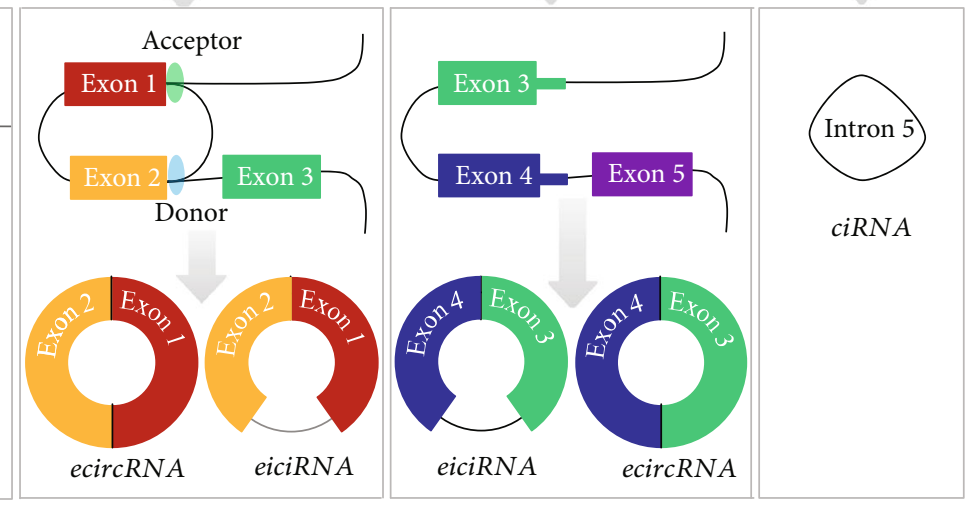

(c)

(d)

(e)

FIgURE 1: The main classification of circRNA. (a) With the interaction of TAFs (trans-acting factors), the cross-exon interactions are turned into cross-intron interactions and lead to the production of liner mRNA. (b) In the process of exon skipping, the cross-exon interactions are retained and ecircRNAs are formed. (c) The combination of the donor splice site and the acceptor splice site promotes the exon skipping. (d) Intron pairing is based on the interaction of complementary sequences or RBPs and their corresponding response elements in the long flanking intron; EIciRNAs, ecircRNAs, and ciRNAs are produced according to whether or not the introns are removed. (e) The C-rich and GU-rich elements present on opposite ends of the intron enhance the stability of intron and promote the formation of ciRNAs.

Recent studies have shown that circular RNA (circRNA) is a type of endogenous noncoding RNA with a stable closed loop structure that can function as a "sponge" for microRNA (miRNA) [11, 12]. Because miRNAs are involved in the development and progression of tumors, circRNAs regulating the pool of miRNAs can also be implicated in tumorigenesis. In addition, it has been shown that there is a significant difference in circRNA levels between GC tissue and adjacent noncancerous tissue. These findings suggest that circRNAs may potentially serve as novel biomarkers for GC.

This review comprehensively and concisely summarizes the classification and function of circRNAs and provides some recent findings on the emerging roles of circRNAs in GC. Finally, their potential use as diagnostic, prognostic, and therapeutic targets in GC is also discussed based on new findings from recent studies.

\section{Classification of circRNAs}

circRNAs can be classified into four types based on their different components and origin: circular exon RNAs (ecircRNAs), circular intronic RNAs (ciRNAs), exon-intron circRNAs (EIciRNAs), and transfer RNA intronic circRNAs (tricRNAs) (Figure 1).

2.1. ecircRNAs. Generally speaking, the generation of ecircRNAs can follow two paths, driven by exon skipping and intron pairing mechanisms (Figure 1). Exon skipping means that the donor splice site of the $3^{\prime}$ joins to the acceptor splice site of the $5^{\prime}$ skipped exon during pre-mRNA splicing, and finally, a head-to-tail circular structure is formed [13]. This phenomenon is often accompanied by the alternative splicing of pre-mRNA and is associated with the existence of core spliceosome components [13-15]. Exon skipping can lead to the production of single- or multiple-exon circles, which are related to the sites of splicing factors $[13,14]$. In the canonical splicing process, splicing complex U1 and U2 small nuclear ribonucleoproteins (snRNPs) will bind downstream and upstream of each exon to build cross-exon interactions. With the interaction of trans-acting factors (such as SR proteins or heterogeneous nuclear ribonucleoproteins), these cross-exon interactions are promoted to turn into cross-intron interactions, which are essential to remove introns and build linear mRNA $[15,16]$. Therefore, the activities of these trans-acting factors influence the levels of circRNAs and their related linear mRNAs.

The occurrence of intron pairing-driven circularization is based on intronic complementary pairing of flanking intron sequences such as Alu elements and RNA-binding proteins (RBPs), which can facilitate the formation of ecircRNAs in the mammalian pre-mRNA splicing system after removal of discontinuous intron sequences [16-19]. Several studies have verified that circularized exons have higher complementary Alu density in their long flanking introns than controls, and these Alu repeats can form inverted repeat Alu element (IRAlu) pairs to promote back-splicing [19, 20]. The intron pairing mechanism is not limited to IRAlu pairs, as other complementary sequences or even some RBPs (e.g., muscleblind (MBL) and quaking) can effectively influence the formation of ecircRNAs $[17,18,20]$. 


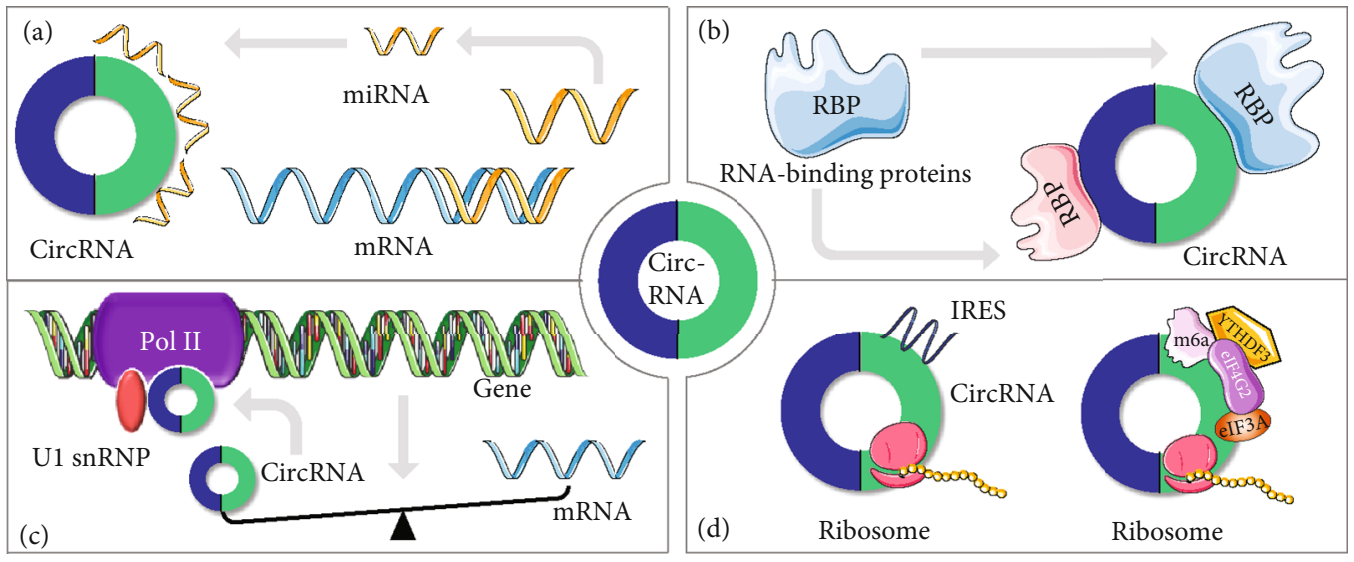

FIgURE 2: Function of circRNAs. (a) circRNAs competitively bind with miRNA which results in less mRNA degradation or translation inhibition. (b) circRNAs interact with RBPs to influence GC progression. (c) circRNAs interact with pol II and U1snRNP to regulate transcription and splicing. (d) circRNAs which encode proteins rely on the IRES-dependent or m6a-dependent translation initiation.

2.2. ciRNAs. ciRNAs, also known as stable intronic sequence RNAs or full-length intronic circularized RNAs, are another class of recently found circRNAs. It is well known that RNase $\mathrm{R}$ can degrade linear RNAs and Y-structure RNAs but has no effect on the loop portion of a lariat RNA [21]. In general, escape from the debranching of intron lariats is the basic reason for ciRNA generation. Several critical sequences contribute to intron circularization including the 7 nucleotide (nt) GU-rich element near the $5^{\prime}$ splice site, the $11 \mathrm{nt}$ C-rich element close to the $3^{\prime}$ branchpoint, and the RNA lariat debranching enzyme Dbr1 element [21, 22]. Among these three elements, the $5^{\prime}$ ss-GU exhibits the best promotion effect for intron circularization; however, Dbr1 might play an indirect role or work together with other factors in this process [22]. In addition, RNA sequences near the $5^{\prime}$ splice site and $\mathrm{C}$ branchpoint can promote inefficient debranching, thereby enhancing the stability of ciRNAs. These key RNA elements are not abundant in regular introns or other types of circRNAs, indicating their significance in allowing an intron lariat to escape debranching $[21,22]$.

2.3. EIciRNAs. In EIciRNAs, introns between circularized exons are retained. EIciRNAs are primarily localized in the nucleus and associated with Pol II, suggesting that they might participate in transcriptional regulation. Indeed, several mechanisms have been proposed to explain their regulatory role in transcription, including enhancement of their parental gene expression in cis. It has been shown that EIciRNAs (circEIF3J and circPAIP2) can serve as potential trans-regulators of nonparental gene loci and even interact with U1 snRNP and Pol II through RNA-RNA interactions [23]. Similar findings were presented by $\mathrm{Hu}$ and Zhou [24] in a theoretical mechanistic gene model, showing that EIciRNAs can notably mediate mRNA and further protein expression.

2.4. tricRNAs. In Archaea and Eukarya, after cleavage by the transfer RNA (tRNA) splicing endonuclease complex, tRNA precursors are split into three parts: linear intron, tRNA $3^{\prime}$ -halves beginning with a $5^{\prime}$-hydroxyl and $5^{\prime}$-halves ending in a $2^{\prime}, 3^{\prime}$-cyclic phosphate. Then, tRNA ligase interacts with the last two parts to build a mature tRNA $[25,26]$. The linear intron fragment is also ligated by ligase RtcB in a head-to-tail form, thus generating stable circRNAs termed tricRNAs [27]. Currently, endogenous human tricRNAs (which are circular and only 16-21 nt long) cannot be verified, as they are excluded from typical RNA sequencing cDNA libraries. However, human cells have all of the necessary machinery to produce tricRNAs [27], which have also been identified in many human cells such as HeLa cervical cancer and human embryonic kidney 293T cells [26].

\section{Mechanisms of circRNA Influence on GC Progression}

The formation of malignant tumors is closely correlated with aberrant cell proliferation, migration, and invasion. An increasing number of studies indicate that circRNAs are able to influence the biological behavior of GC cells by mediating cell cycle- or migration-related factors. Although some of their mechanisms of action remain to be clarified, the proven mechanisms are summarized below and in Figure 2.

3.1. circRNAs Acting as miRNA Sponges. The most common mechanism by which circRNAs participate in cancer progression is by acting as miRNA sponges, also known as competing endogenous RNAs (ceRNAs). In this way, they influence the posttranscriptional regulation of gene expression. Some circRNAs have more than one miRNA-binding site, which has been confirmed by several studies [12, 28, 29]. For example, CDRlas (antisense to the cerebellar degeneration-related protein 1 transcript), also named ciRS7 (circRNA sponge for miR-7), contains more than 70 miRNA seed regions for miR-7, which matches and binds Argonaute proteins (AGO), allowing it to function as an excellent regulator of miR-7 $[12,28]$. The zebrafish embryonic brain lacks the CDR1 locus, but steadily and highly expresses miR-7 [12, 30]. When CDR1as RNA was introduced into this model, consistent impairment of the 
TABLE 1: circRNA-miRNA-mRNA/protein network in GC.

\begin{tabular}{|c|c|c|c|c|c|c|}
\hline Circular RNAs & Expression & $\begin{array}{l}\text { Parental } \\
\text { gene }\end{array}$ & $\begin{array}{l}\text { Sponged } \\
\text { miRNA }\end{array}$ & Targeted mRNA/protein & Related cell behaviors & Ref \\
\hline hsa_circ_0006282 & Up & 1 & miR-155 & $\mathrm{FBXO} 22$ & Proliferation, metastasis & [105] \\
\hline hsa_circ_000684 & Up & l & $\operatorname{miR}-186$ & ZEB1 & $\begin{array}{l}\text { Proliferation, migration, } \\
\text { invasion, tube formation }\end{array}$ & [106] \\
\hline hsa_circRNA_001569 & Up & l & $\operatorname{miR}-145$ & NR4A2 & Proliferation, apoptosis, & [107] \\
\hline hsa_circRNA_102958 & $\mathrm{Up}$ & l & miR-585 & CDC25B & Proliferation, migration, invasion, & [108] \\
\hline hsa_circRNA_104433 & $\mathrm{Up}$ & ARPC1B & miR-497-5p & CDC25A & Proliferation, apoptosis & [109] \\
\hline hsa_circ_0000291 & Up & CD44 & miR-183 & ITGB1 & Proliferation, migration & [110] \\
\hline hsa_circ_0017639 & Up & SFMBT2 & miR-224-5p & USP3 & Proliferation, migration & [111] \\
\hline hsa_circ_0092306 & Up & $\operatorname{chr} 11$ & miR-197-3p & PRKCB & $\begin{array}{l}\text { Proliferation, apoptosis, } \\
\text { migration, invasion, }\end{array}$ & [112] \\
\hline Circ-ATAD1 & Up & ATAD1 & miR-140-3p & YY1/PCIF1 & $\begin{array}{l}\text { Proliferation, apoptosis, } \\
\text { migration, invasion }\end{array}$ & {$[88]$} \\
\hline Circ-ATXN7 & Up & ATXN7 & $\operatorname{miR}-4319$ & ENTPD4 & Proliferation, apoptosis, migration & [113] \\
\hline circ-PIP5K1A & Up & PIP5K1A & miR-671-5p & KRT80 & $\begin{array}{l}\text { Proliferation, migration, } \\
\text { invasion, EMT process }\end{array}$ & {$[34]$} \\
\hline circ-ERBB2 & Up & ERBB2 & miR-503,miR-637 & CACUL1, MMP-19 & $\begin{array}{l}\text { Proliferation, apoptosis, } \\
\text { migration, invasion }\end{array}$ & {$[46]$} \\
\hline circ-NHSL1 & $\mathrm{Up}$ & NHSL1 & $\operatorname{miR}-1306-3 p$ & SIX1/vimentin & Migration, invasion & {$[45]$} \\
\hline circ-PVT1 & Up & PVT1 & miR-125b & E2F2 & Proliferation & {$[43]$} \\
\hline circ-HIPK3 & Up & HIPK3 & $\operatorname{miR}-124 / 29 b$ & COL1A1/COL4A1/CDK6 & Proliferation & {$[41]$} \\
\hline circ-OSBPL10 & Up & OSBPL10 & miR-136-5p & WNT2 & Proliferation, migration, invasion & {$[40]$} \\
\hline circ-NOTCH1 & Up & NOTCH & $\operatorname{miR}-449 c-5 p$ & MYC/NOTCH1 & Proliferation, migration, invasion & {$[42]$} \\
\hline circ-NRIP1 & Up & NRIP1 & miR-149-5p & AKT1/mTOR pathway & Proliferation, migration, invasion & {$[36]$} \\
\hline ciRS-7 & Up & Cdr1as & miR-7 & PTEN/PI3K/AKT pathway & Proliferation, migration, invasion & {$[32]$} \\
\hline circ-MAN2B2 & Up & MAN2R2 & miR-145 & PI3K/AKT/JNK pathway & Proliferation, migration & {$[35]$} \\
\hline circ-HIAT1 & Down & HIAT1 & $\operatorname{miR}-21$ & $\begin{array}{c}\text { PTEN/PI3K/AKT/ERK } \\
\text { pathway }\end{array}$ & $\begin{array}{l}\text { Proliferation, apoptosis, } \\
\text { EMT process }\end{array}$ & {$[33]$} \\
\hline circ-ZFR & Down & ZFR & miR-130a/107 & PTEN & Proliferation, apoptosis & {$[38]$} \\
\hline circ-YAP1 & Down & YAP1 & miR-367-5p & p27 Kip1 & Proliferation, invasion & {$[114]$} \\
\hline circ-RHOBTB3 & Down & RHOBTB3 & miR-654-3p & p21 & Proliferation, apoptosis & [115] \\
\hline circ-LARP4 & Down & LARP4 & $\operatorname{miR}-424-5 p$ & LATS1 & Proliferation, invasion & {$[90]$} \\
\hline circ-FAT1 (e2) & Down & FAT1 & miR-548g & RUNX1 & Proliferation, invasion, migration. & {$[52]$} \\
\hline circ-CCDC9 & Down & CCDC9 & miR-6792-3p & CAV1 & Proliferation, migration, invasion & [116] \\
\hline hsa_circ_0001368 & Down & KLHL24 & miR-6506-5p & FOXO3 & Proliferation, invasion & [117] \\
\hline
\end{tabular}

zebrafish midbrain was observed, similar to the effect of miR-7 inhibition [12].

This mechanism can be widely found in GC-related circRNAs (Table 1). Through binding to miRNA, they can regulate tumor-related canonical signaling pathways. The phosphatase and tensin homolog (PTEN)/phosphatidylinositol 3-kinase (PI3K)/protein kinase B (AKT) pathway is a critical regulator of cellular activities, in which PTEN acts as a tumor suppressor that inhibits cell proliferation and metastasis [31]. CiRS-7 is overexpressed in GC tissues and is closely correlated with some malignant clinicopathological features of GC. Indeed, the overexpression of CiRS-7 antagonizes the level of miR-7, which leads to decreased PTEN and increased PI3K and AKT phosphorylation, eventually resulting in the oncogenic effects of the PTEN/PI3$\mathrm{K} / \mathrm{AKT}$ pathway [32]. Conversely, circHIAT1 expression is low in GC tissues, and its overexpression suppresses activation of the PTEN/PI3K/AKT and extracellular signalregulated kinase signaling pathways. This effect is achieved through circHIAT1 binding to miR-21, which results in the inhibition of cell proliferation and promotion of apoptosis [33]. Moreover, circPIP5K1A [34], circMAN2B2 [35], circNRIP1 [36], and circNF1 [37] are all upregulated in GC samples and promote GC progression through the miR-671-5p-KRT80-PI3K/AKT, miR-145-PI3K/AKT/JNK, miR-149-5p-AKT/mTOR, and miR-16-AKT axes, respectively. circ-ZFR [38] and circGRAMD1B [39] are distinctly downregulated in GC tissues and exert antitumor effects by inhibiting the miR-130a/miR-107-PTEN/p53 axis and miR130a-3p-PTEN/p21 axis, respectively.

In addition to their influence on tumor-related signaling pathways, circRNAs can also regulate some tumor oncogenes 
or antioncogenes by acting as miRNA sponges. For example, the overexpression of circOSBPL10 has been verified in GC tissues and has been shown to positively correlate with tumor growth, migration, and metastasis both in vitro and in vivo. These tumorigenic actions can be explained by the circOSBPL10-miR-136-5p-WNT2 axis [40]. Similarly, Cheng et al. also found that upregulation of circHIPK3 promotes cell proliferation by sponging miR-124/miR-29b to regulate the human collagen genes (COL1A1, COL4A1) and CDK6 [41]. Furthermore, it has been shown that circNOTCH1 can function as a promoting factor for the migration, invasion, and stemness of tumors by binding to miR-449c-5p to influence Myc expression, further modulating the transcription of NOTCH1 [42]. In addition, circPVT1 possesses two binding sites for miR-125b and, by sponging to miR-125b, can promote expression of the E2F2 gene and facilitate GC cell proliferation [43]. Interestingly, circPVT1 also contributes to the chemoresistance of GC cells through the ceRNA mechanism. It has been shown that circPVT1 is highly regulated in paclitaxel- (PTX-) resistant GC tissues and cells. By attenuating the function of miR-124-3p, the level of zinc finger Ebox binding homeobox 1 (ZEB1) is elevated, thereby leading to the enhanced PTX resistance of GC [44]. Finally, some migration-related proteins, including matrix metalloproteinase (MMP) and epithelial-to-mesenchymal transition-related markers, can be mediated through this mechanism. For instance, circNHSL1 is overexpressed in GC tissues and is positively associated with Union for International Cancer Control/tumor-node-metastasis (TNM) stage. By serving as a miR-1306-3p sponge, the highly expressed circNHSL1 removes the inhibitory effects of miR-1306-3p by acting on SIX1, resulting in increased vimentin protein expression and decreased GC growth and metastasis in vitro and in vivo [45]. Through the circERBB2/miR-637/MMP-19 pathway, circERBB2 positively regulates MMP-19 levels, thereby promoting cell metastasis and invasion [46].

3.2. circRNA Interaction with RBPs. Cyclin-dependent kinase 2 (CDK2), as a member of the serine/threonine (Ser/Thr) kinase family, is a vital regulator of the cell cycle. Through binding to its partner cyclin $\mathrm{E}$ or $\mathrm{A}$, this protein can promote the G1/S transition and DNA replication [47]. However, circFoxo3 is able to interact with CDK2 and p21 (CDK inhibitor 1) protein simultaneously, which thus induced the inhibition of p21 in CDK2 and blocked cell cycle progression [48].

Human antigen $\mathrm{R}(\mathrm{HuR})$ protein is well known for its ability to regulate the stability and translation of labile mRNA, which is achieved by binding to the AU-rich RNA stretches and $3^{\prime}$-untranslated region (UTR) of target mRNA [49]. In GC, circAGO2 can directly interact with HuR protein to facilitate its effect on the $3^{\prime}$-UTR of target genes. This in turn leads to less gene silencing and enhances tumorigenesis and tumor aggressiveness [50]. In addition, circ-HuR serves as an inhibitor of HuR protein by directly interacting with CCHC-type zinc finger nucleic acid binding protein, further resulting in the suppression of HuR expression [51]. Furthermore, circFAT1 (e2) also has the ability to suppress the proliferation, invasion, and migration of GC cells. circFAT1 (e2) can act as a sponge of miR-548g in the cytoplasm to upregulate the RUNX1 expression. This leads to the inhibition of tumorigenesis, and this effect can be accomplished by directly binding to YBX1 protein in the nucleus [52]. Hong et al. [53] reported that increased circFNDC3B promotes the migration and invasion of GC cells and affects the expression of migration-related proteins, including reduced E-cadherin, increased N-cadherin, SNAI1, and vimentin levels, which might be explained by circFNDC3B directly interacting with IGF2BP3 protein leading to the increased CD44 expression.

3.3. circRNAs in the Regulation of mRNA Transcription and Splicing. Ashwal-Fluss et al. [18] reported that the circularization rates of circRNAs strongly depend on the existence of canonical splice sites in bracketing exons, which can reduce the splicing efficiency of the linear transcriptome, indicating the competition between the production of circRNAs and linear splicing. Based on this mechanism, excess MBL protein can promote circMBL production, thus reducing formation of its own mRNA [18]. Regarding transcription regulation, apart from the aforementioned EIciRNAs, some newly discovered circRNAs also have this function. CircMRPS35, as a novel circRNA identified in $\mathrm{GC}$, is negatively related to several poor clinicopathologic factors. Through attracting histone acetyltransferase KAT7, circMRPS35 elevates H4K5 acetylation in the promoter region of FOXO1 and FOXO3a genes, which further promotes the expression of FOXO1 and FOXO3a and eventually triggers the expression of p21, p27, Twist1, and E-cadherin. This process contributes to suppressing the proliferation and invasion of GC cells [54].

3.4. Translation of circRNAs. The canonical translational process is termed cap-dependent translation. In this process, the mRNA $5^{\prime}$-cap structure is an indispensable part, as it identifies the translation initiation complex and initiates protein synthesis [55]. However, circRNAs lack the $5^{\prime}$-cap structure, and as a result, their translation potential was ignored for a long time. In recent years, the cap-independent translation of circRNAs has been identified in eukaryotic cells, and its mechanism is mainly divided into "internal ribosome entry site- (IRES-) dependent initiation" and "N6-methyladenosine- $\left(\mathrm{m}^{6} \mathrm{~A}-\right)$ dependent initiation" at present [56]. Internal ribosome entry site elements can mediate the translation initiation in a cap-independent manner, which were originally found in viruses [56]. The presence of an open reading frame (ORF) and STOP codon in circ-ZNF609 has led researchers to examine its protein-coding ability. During its translation initiation process, the $5^{\prime}$-UTR sequence is able to drive IRES-dependent translation in a spicing-dependent manner; thus, necessary factors to activate IRES elements can be loaded on a correct splicing event [57]. A similar mechanism can also be verified for circSHPRH [58] and circLINC-PINT [59], since their IRES activity is distinctly associated with the existence of a backsplicing junction. N6-methyladenosine $\left(\mathrm{m}^{6} \mathrm{~A}\right)$ is widely present in eukaryotes, and its translationpromoting effect was also confirmed in mRNA and circRNA $[56,60]$. Yang et al. [61] found that with the participation of initiation factor eIF4G2 and eIF3A, as well as m6A reader 
YTHDF3, m6A modification effectively facilitated the translation initiation of circRNAs in human cells. Furthermore, $\mathrm{m}^{6} \mathrm{~A}$-dependent translation initiation could be promoted or inhibited by methyltransferase or demethylase [61].

Although the function of circRNA-derived protein remains ambiguous, protein-coding ability has been found in some GC-related circRNAs. The circRNA database (circRNADb) indicates that circFNDC3B possesses a potential IRES and ORF. In addition, it has been shown that circFNDC3B can encode a peptide of approximately $25 \mathrm{kDa}$ [53]. Besides, circPVRL3 contains IRES, ORF, and m6A modification structures, which provide a potential for its translation [62].

\section{Applications of circRNA in GC}

4.1. circRNAs for GC Screening and Diagnosis. Chronic gastric diseases including gastric polyps, chronic atrophic gastritis, and gastric stump after partial gastrectomy often precede the development of GC. Studies have shown that many circRNAs are differentially expressed in cells, tissues, and even the bodily fluids of people with GC, precancerous gastric lesions, and healthy individuals $[63,64]$. circRNAs are quite stable in clinical samples and are associated with several GC clinicopathological factors. For some, their diagnostic value is even higher than existing diagnostic markers such as carcinoembryonic antigen (CEA) and carbohydrate antigen 19-9 (CA 19-9) [65-68], raising the possibility of using circRNAs to diagnose GC and evaluate patients' prognosis. Moreover, some circRNAs have differential expression in the plasma and gastric juice of patients compared to healthy individuals and have potential to be used as noninvasive GC diagnostic biomarkers [69, 70] (Table 2).

To date, three types of upregulated circRNAs, namely, hsa_circ_0066444 [71], hsa_circRNA_102958 [72], and hsa_circ_0000467 [66], have been identified in GC tissues that can act as diagnostic biomarkers. Their reported AUC values are $0.7328,0.74$, and 0.79 , respectively. After analyzing the clinicopathological characteristics of GC patients and their tumors with respect to hsa_circ_0066444 expression in 106 GC samples, a positive association was found between its expression and lymph node metastasis [71].

Apart from these overexpressed circRNAs, multiple downregulated circRNAs with potential diagnostic value have been confirmed in GC tissues or blood samples. Among them, seven circRNAs have been verified both in tissues and in plasma including hsa_circ_0000190 [67], hsa_circ_ 0000181 [73], hsa_circ_0000520 [74], hsa_circ_0001821 [68], hsa_circ_0001017, hsa_circ_0061276 [75], and hsa_ circ_0000419 [76]. The last five had higher AUC values above $0.84(0.89,0.87,0.851,0.849$, and 0.840 , respectively) in plasma. The AUC values for all circRNAs except hsa_ circ_0000520 and hsa_circ_0000419 in tissue samples were not less than $0.75,(0.75,0.756,0.792,0.871$, and 0.764 , respectively). Furthermore, the combination of two plasma circRNAs hsa_circ_0001017 and hsa_circ_0061276 increased the AUC value to 0.912 with $84.7 \%$ sensitivity and $96.6 \%$ specificity [75]. In addition, when both the plasma and tissue levels of hsa_circ_0001017 and hsa_circ_0061276 were used in combination, the AUC value reached 0.966 with a sensitivity and specificity of $95.5 \%$ and $95.7 \%$, respectively [75]. Collectively, these results show the great potential of using circRNAs as biomarkers for GC diagnosis and highlight the necessity of using integrated biomarkers to elevate their diagnostic efficiency. Regarding the clinical correlation analysis, both hsa_circ_0000190 and hsa_circ_ 0000181 expressions in tissues were negatively associated with tumor size, CA19-9 levels, and lymphatic and distal metastases [67, 73]. The clinical values of hsa_circ 0006848 and hsa_circ_0000745 were only explored in the plasma of a smaller cohort of GC patients, and their AUC values were 0.733 and 0.683 , respectively $[77,78]$.

In addition to those previously mentioned, 11 other circRNAs analyzed in GC tissues showed promising diagnostic potential. Among these, hsa_circ_0005654 [79], hsa_circ_ 00001649 [80], hsa_circ_0000096 [65], hsa_circ_0001895 [81], and hsa_circ_0003159 [82] had better diagnostic usefulness with AUC values of $0.927,0.834,0.82,0.792$, and 0.75 , respectively. Combined with hsa_circ_0000096 and hsa_ circ_002059, the AUC value rose to 0.91 [65]. The expression of hsa_circ_00001649 was associated with tumor differentiation, but hsa_circ_0000096 and hsa_circ_0003159 expression was associated with gender, distal metastasis, and TNM stage $[65,80,82]$. The hsa_circ_0001895 is also correlated with tumor differentiation, as well as with Borrmann type and tissue CEA expression [81].

Another six circRNAs including hsa_circ_0067582, hsa_ circ_0005758 [83], hsa_circ_00014717 [11], hsa_circ_ 002059 [84], hsa_circ_0006633 [85], and hsa_circ_0130810 [86] have a lower diagnostic value with AUCs of 0.671, $0.721,0.696,0.73,0.741$, and 0.748, respectively. Among these, hsa_circ_0014717 is remarkably downregulated in $77.2 \%$ (74/96) GC tissues. In addition, it was also relatively stable in human gastric juice and its level in chronic atrophic gastritis patients was even lower than that in GC patients. These findings suggest that it can be used as a gastric juice biomarker for GC [11]. Finally, a meta-analysis of circRNA and GC by Jiang et al. [63] recently showed that although the number of studies examining circRNAs in GC plasma or saliva is limited, circRNAs have potential use as specific and accurate diagnostic biomarkers, with overall sensitivity, specificity, and AUC above $70 \%$.

4.2. circRNAs in GC Prognosis. TNM stage is the main prognostic factor for the prediction of overall survival (OS) in GC patients. Nevertheless, with the emerging precision medicine and targeted therapy, more accurate and specific prognostic indicators are needed to choose the most appropriate treatment, hopefully resulting in better outcomes.

Several studies have reported the association between circRNA and GC patient prognosis (Table 3). Recently, the predictive potential of 12 circRNAs has been reported in tissues. Eight of these circRNAs were upregulated including hsa_circ_0010882 [87], hsa_circRNA_ATAD1 [88], hsa_circRNA_PVT1 [43], hsa_circRNA_HIPK3 [41], hsa_ circRNA_DCAF6 [89], hsa_circRNA_OSBPL10 [40], hsa_ circRNA_NHSL1 [45], and hsa_circRNA_NRIP1 [36], 
TABle 2: Diagnostic efficiency of circRNAs in GC.

\begin{tabular}{|c|c|c|c|c|c|c|c|c|c|}
\hline \multirow{2}{*}{ circRNAs } & \multirow{2}{*}{ Samples } & \multirow{2}{*}{ Clinical association } & \multicolumn{4}{|c|}{ Diagnostic value } & \multicolumn{2}{|c|}{ Sample size } & \multirow{2}{*}{ Ref } \\
\hline & & & Sens & Spec & AUC & Cutoff value & Case & Control & \\
\hline \multicolumn{10}{|l|}{ Oncogenic } \\
\hline hsa_circ_0066444 & Tissues & Lymphatic metastasis & 0.7075 & 0.6887 & 0.7328 & - & 106 & 106 & [71] \\
\hline hsa_circRNA_102958 & Tissues & TNM stage & 0.61 & 0.86 & 0.74 & - & 30 & 30 & {$[72]$} \\
\hline hsa_circ-0000467 & Tissues & TNM stage & 0.705 & 0.648 & 0.790 & - & 51 & 51 & [66] \\
\hline \multicolumn{10}{|l|}{ Antioncogenic } \\
\hline hsa_circ_0067582 & Tissues & Tissue CEA level and stages & 0.552 & 0.750 & 0.671 & 10.61 & 96 & 96 & [83] \\
\hline hsa_circ_0005758 & & CEA level and perineural invasion & 0.750 & 0.677 & 0.721 & 10.20 & & & \\
\hline hsa_circ_0003159 & Tissues & Gender, distal metastasis, and TNM stage & 0.852 & 0.565 & 0.75 & 12.31 & 108 & 108 & [82] \\
\hline hsa_circ_0005654 & Tissues & - & - & - & 0.927 & - & 68 & 68 & [79] \\
\hline hsa_circ_00001649 & Tissue & Pathological differentiation & 0.711 & 0.816 & 0.834 & 0.2269225 & 76 & 76 & [80] \\
\hline hsa_circ_0000096 & Tissues & Gender, invasion, and TNM stage & 0.88 & 0.56 & 0.82 & 12.9 & 101 & 101 & [65] \\
\hline hsa_circ_0014717 & Tissues & $\begin{array}{l}\text { Tumor stage, distal metastasis, tissue } \\
\text { CEA, and CA19-9 expression }\end{array}$ & 0.5938 & 0.8125 & 0.696 & 12.14 & $96 \mathrm{GC}$ & 96 & [11] \\
\hline hsa_circ_0001895 & Tissues & $\begin{array}{l}\text { Tumor differentiation, Borrmann type, } \\
\text { and tissue CEA expression }\end{array}$ & 0.678 & 0.857 & 0.792 & 9.53 & $96 \mathrm{GC}$ & 96 & [81] \\
\hline hsa_circ_002059 & Tissues & $\begin{array}{c}\text { Distal metastasis, TNM stage, gender, } \\
\text { and age }\end{array}$ & 0.81 & 0.62 & 0.73 & 12.9 & 101 & 101 & [84] \\
\hline hsa_circ_0006633 & Tissues & Distal metastasis and tissue CEA level & 0.60 & 0.81 & 0.741 & 8.17 & 96 & 96 & [85] \\
\hline hsa_circ_0130810 & Tissues & TNM stage and lymphatic metastasis & 0.7742 & 0.6800 & 0.7481 & 1.443 & 28 & 28 & [86] \\
\hline hsa_circ_0006848 & Plasma & Tumor differentiation and tumor size & - & - & 0.733 & - & 30 & 30 & [77] \\
\hline hsa_circ_0000745 & Plasma & TNM stage & 0.855 & 0.45 & 0.683 & & 60 & 60 & [78] \\
\hline \multirow[t]{2}{*}{ hsa_circ_0000190 } & Tissues, & $\begin{array}{l}\text { Tumor diameter, lymphatic and distal } \\
\text { metastasis, TNM stage, and CA19-9 }\end{array}$ & 0.721 & 0.683 & 0.75 & 6.83 & 104 & 104 & [67] \\
\hline & Plasma & CEA & 0.414 & 0.875 & 0.60 & 3.07 & 104 & 104 & \\
\hline \multirow[t]{2}{*}{ hsa_circ_0000181 } & $\begin{array}{l}\text { Tissues, } \\
\text { plasma }\end{array}$ & $\begin{array}{l}\text { Tumor size, lymphatic metastasis, distal } \\
\text { metastasis, and CA19-9 }\end{array}$ & 0.990 & 0.852 & 0.756 & 9.40 & 115 & 115 & [73] \\
\hline & & Tumor differentiation and CEA level & $99.0 \%$ & $20.6 \%$ & 0.582 & 7.27 & 102 & 105 & \\
\hline \multirow{2}{*}{ hsa_circ_0000520 } & Tissues, & TNM stage & 0.5357 & 0.8571 & 0.6129 & - & 56 & 56 & [74] \\
\hline & Plasma & CEA expression & 0.8235 & 0.8444 & 0.8967 & - & 45 & 17 & \\
\hline \multirow{2}{*}{ hsa_circ_0001821 } & Tissues, & Tumor depth and lymph node metastasis & - & - & 0.792 & - & 80 & 80 & [68] \\
\hline & Plasma & - & - & - & 0.872 & - & 30 & 30 & \\
\hline \multirow{2}{*}{ hsa_circ_0001017 } & Tissues, & $\begin{array}{l}\text { Age, tumor size, invasion, TNM stages, } \\
\text { distal metastasis, and CEA levels }\end{array}$ & 0.794 & 0.811 & 0.871 & - & 121 & 121 & [75] \\
\hline & Plasma & $\begin{array}{l}\text { Gender, tumor size, differentiation, } \\
\text { and distal metastasis }\end{array}$ & 0.676 & 0.897 & 0.851 & - & & & \\
\hline \multirow{2}{*}{ hsa_circ_0061276 } & Tissues, & $\begin{array}{l}\text { Age, tumor size, TNM stages, distal } \\
\text { metastasis, and CEA levels }\end{array}$ & 0.913 & 0.507 & 0.764 & - & & & \\
\hline & Plasma & $\begin{array}{l}\text { Gender, tumor size, differentiation, } \\
\text { and CEA levels }\end{array}$ & 0.758 & 0.959 & 0.849 & - & & & \\
\hline \multirow[b]{2}{*}{ hsa_circ_0000419 } & Tissues, & Borrmann type and differentiation grade & 0.552 & 0.677 & 0.642 & 8.14 & 96 & 96 & \multirow[t]{2}{*}[76]{} \\
\hline & Plasma & $\begin{array}{l}\text { Tumor stage, lymphatic and distal } \\
\text { metastasis, venous and perineural invasion }\end{array}$ & 0.682 & 0.884 & 0.840 & 4.9 & 44 & 43 & \\
\hline
\end{tabular}

Sens: sensitivity; Spec: specificity.

whereas 4 were downregulated including hsa_circRNA_ LARP4 [90], hsa_circRNA_LMTK2 [91], hsa_circRNA_ PVRL3 [62], and hsa_circRNA_CCDC9 [45]. In addition, a four-circRNA-based classifier with hsa_circRNA_101308 (upregulated), hsa_circRNA_104423 (downregulated), hsa_ circRNA_104916 (downregulated), and hsa_circRNA_ 100269 (downregulated) involved was also identified.
Among these eight upregulated circRNAs (hsa_circ 0010882 [87], hsa_circRNA_ATAD1 [88], hsa_circRNA_ PVT1 [43], hsa_circRNA_HIPK3 [41], hsa_circRNA_ DCAF6 [89], hsa_circRNA_OSBPL10 [40], hsa_circRNA_ NHSL1 [45], and hsa_circRNA_NRIP1 [36]), Kaplan-Meier analysis indicated that GC patients with high circRNA levels tend to have poorer OS, while low expression of circPVT1, 
TABle 3: Prognosis-related circRNAs in GC.

\begin{tabular}{|c|c|c|c|c|c|c|}
\hline circRNAs & $\begin{array}{l}\text { Number of } \\
\text { patients }\end{array}$ & Samples & Dysregulation & Correlated clinicopathologic features & Clinical values & Ref \\
\hline hsa_circ_0010882 & 49 & Tissues & Upregulated & TNM stage and tumor size & Poor OS & [87] \\
\hline circ-ATAD1 & 72 & Tissues & Upregulated & $\begin{array}{l}\text { Tumor size, invasion, lymphatic } \\
\text { metastasis, TNM stage }\end{array}$ & Poor OS & {$[88]$} \\
\hline circ-HIPK3 & 63 & Tissues & Upregulated & T stage and Ming's classification & Poor OS & {$[41]$} \\
\hline circ-DCAF6 & 62 & Tissues & Upregulated & - & Poor OS & [89] \\
\hline circPVT1 & 187 & Tissues & Upregulated & - & $\begin{array}{l}\text { Poor OS } \\
\text { and DFS }\end{array}$ & {$[43]$} \\
\hline circ-OSBPL10 & 70 & Tissues & Upregulated & $\begin{array}{c}\text { T stage, TNM stage, and tumor location } \\
\text { (cardia GC show higher expression than } \\
\text { noncardia GC) }\end{array}$ & $\begin{array}{l}\text { Poor OS } \\
\text { and DFS }\end{array}$ & {$[40]$} \\
\hline circ-NHSL1 & 93 & Tissues & Upregulated & $\begin{array}{c}\text { UICC stages, pathological T stages, } \\
\text { lymphatic metastasis, distant metastasis, } \\
\text { and grades }\end{array}$ & $\begin{array}{l}\text { Poor OS } \\
\text { and DFS }\end{array}$ & {$[45]$} \\
\hline circ-NRIP1 & 110 & Tissues & Upregulated & Tumor size, lymphatic invasion & $\begin{array}{l}\text { Poor OS } \\
\text { and DFS }\end{array}$ & {$[36]$} \\
\hline circ-LARP4 & 387 & Tissues & Downregulated & Tumor size and lymphatic metastasis & Better OS & {$[90]$} \\
\hline circ-LMTK2 & 111 & Tissues & Downregulated & TNM stage and lymphatic metastasis & Better OS & {$[91]$} \\
\hline circ-PVRL3 & 62 & Tissues & Downregulated & TNM stage & Better OS & {$[62]$} \\
\hline circ-CCDC9 & 48 & Tissues & Downregulated & $\begin{array}{l}\text { Tumor size, lymph node invasion, } \\
\text { and TNM stage }\end{array}$ & Better OS & [116] \\
\hline $\begin{array}{l}\text { Four-circRNA- } \\
\text { based classifier }\end{array}$ & 125 & Tissues & 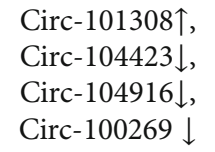 & $\begin{array}{l}\text { Effectively predict the early recurrence } \\
\text { of stage III GC. (with HR value and } \\
\text { ROC curve were provided) }\end{array}$ & $\begin{array}{l}\text { Recurrence- } \\
\text { related biomarker }\end{array}$ & [92] \\
\hline
\end{tabular}

circRNA_OSBPL10, circRNA_NHSL1, and hsa_circRNA_ NRIP1 was also associated with lower disease-free survival (DFS) $[36,40,43,45]$. Furthermore, univariate and multivariate Cox proportional hazards analyses revealed that overexpression of circ-DCAF6 and circPVT1 is a risk factor for GC patient survival. In addition, they are independent prognostic factors for OS and disease-free survival (DFS) in GC patients $[43,89]$. Meanwhile, most of these circRNAs are associated with TNM stages and lymphatic metastasis. Downregulated circRNAs including hsa_circRNA_LARP4, hsa_circRNA_ LMTK2, hsa_circRNA_PVRL3, and hsa_circRNA_CCDC9 were positively correlated with GC patient survival time, and their independent predictive potential for OS was verified through the univariate and multivariate Cox proportional hazard analyses [62, 90, 91]. The TNM stage was negatively associated with the expression of circPVRL3, circLTMK2, and circCCDC9 $[62,91]$, while a higher incidence of lymphatic metastasis was found in patients whose tumors were presented with lower circ-LARP4, circLTMK2, and circCCDC9 level [90, 91].

By examining the circRNA expression profile of 125 pairs of cancer and adjacent normal tissues of stage III GC patients, Zhang et al. [92] established a four-circRNA-based classifier including hsa_circRNA_101308, hsa_circRNA_ 104423, hsa_circRNA_104916, and hsa_circRNA_100269 in the recurrence forecast model of stage III GC and confirmed its sensitivity and specificity. In brief, patients were separated into the high-risk and low-risk groups by the cutoff value calculated using this model. According to their results, the recurrence rates in the low-risk and highrisk groups were $15.6 \%$ and $68.2 \%$, respectively, while the AUC values were 0.763 and 0.711 , respectively. In addition, when combined with traditional TNM stages, the AUC value of these two cohorts was 0.866 and 0.818 , respectively [92]. Collectively, these results suggest that patients' recurrence rates can be accurately calculated by constructing the model of early recurrence and even further improved through combining circRNAs with traditional TNM stages.

4.3. circRNAs in GC Treatment. Chemotherapy as an indispensable adjuvant treatment strategy has been routinely applied to the advanced stage GC. To effectively suppress tumor growth and metastasis, some combination regimens can simultaneously restrain several oncogenic pathways which are recommended to use [93]. However, how to alleviate the chemotherapy resistance and toxicity has become an urgent issue in order to provide better treatment of GC patients. CircRNAs can influence radioresistance and chemoresistance through mediating drug accumulation, DNA repair, autophagy, target gene amplification, and the tumor microenvironment [94]. In GC, circAKT3 (hsa_circ_0000199) is highly expressed in cisplatin- (CDDP-) resistant GC samples and associated with multiple aggressive features including tumor size, histological grade, clinical stage, $\mathrm{T}$ classification, and CDDP resistance. Through the circAKT3/miR-198/PIK3R1 axis, circAKT3 distinctly improved the tolerance of GC cells to CDDP, leading to poor DFS [95]. Similarly, circFN1 suppressed the apoptosis of CDDP-resistant cell lines by 
sponging the miR-182-5p and eventually enhancing the CDDP resistance [96]. The paclitaxel (PTX) resistance of GC can be facilitated through the circPVT1/miR-124-3p/ZEB1 axis [44]. Hsa_circ_0001546 binds to miR-421, resulting in the activation of the ATM/Chk2/p53-dependent pathway, which in turn reduces the oxaliplatin $(\mathrm{L}-\mathrm{OPH})$ resistance of HGC-27 cells [97]. In addition, autophagy activation, which is mediated by the promotion of the circRACGAP $1 / \mathrm{miR}$ 3657/ATG7 axis, alleviates apatinib-induced cell apoptosis and reduces the sensitivity of GC to apatinib treatment [98].

Exosomes, as small membrane vesicles, are secreted from various cell types and usually act as intercellular communicators. Tumor cells secrete exosomes, which function as a communication bridge between tumor cells and surrounding cells [99]. Several GC-related circRNAs have been found in exosomes. For instance, circNRIP1 and circ-RanGAP1 promote GC progress via the miR-149-5p/AKT1 axis and miR-877-3p/VEGFA axis, respectively. In previous studies, when their exosomal form was injected into the vein of model animals in vivo or incubated with GC cells in vitro, the enhanced tumor metastasis and migration were observed $[36,100]$. Because circRNAs participate in GC progression through several mechanisms mediating the expression of tumor-related target genes or proteins and can be transported by exosomes, introducing exosomes containing circRNAs into target-therapy for tumor might have a promising future.

\section{Conclusion and Perspective}

circRNAs, as their name suggests, are equipped with a covalently closed structure and previously thought to be useless byproducts of aberrant splicing. However, there is now accumulating evidence that this new type of RNAs is characterized by various expression patterns, complicated regulatory networks, and emerging roles at multiple molecular levels including miRNA, mRNA, and protein. [101]. Current studies have revealed that circRNAs regulate the proliferation and migration of GC cells through several mechanisms. In addition, they have the potential to work as suitable diagnostic or prognostic biomarkers and even effective therapeutic targets. However, some issues still deserve attention to provide a better understanding of GCrelated circRNAs and apply these novel molecular targets clinically as soon as possible.

First, most circRNAs modulating GC cell proliferation and progression are limited to serving as miRNA sponges. Although only a few of them have been found to interact with RBPs or regulate transcription directly, it is unknown whether circRNA-derived proteins participate in GC progress. Second, m6A as a widespread modification in eukaryotes, is able to mediate circRNA translation, but it is unknown if there are any other modifications in circRNAs that mediate the degradation of circRNAs. Third, it is well known that BCR/ABL fusion gene has been used for the diagnosis and treatment of chronic myelogenous leukemia. Similarly, circRNAs generated from fusion genes (f-circRNAs) as a new type of circRNAs also correlate with tumor progression. Guarnerio et al. [102] found that f-circRNAs can be generated from chromosomal translocations in various tumor types. Moreover, f-circM9 (derived from MLL/AF9 fusion gene) not only is capable of promoting cell proliferation and leukemia progression but also results in resistance to therapy. Similar results in non-small-cell lung cancer (NSCLC) have shown that both F-circSR [103] and FcircEA-2a [104], originating from the SLC34A2-ROS1 and EML4-ALK fusion gene, respectively, promote the migration of NSCLC cells. Moreover, it is possible that F-circEA-2a can serve as a novel "liquid biopsy" diagnosis biomarker for EML4-ALK-positive NSCLC. Therefore, it is worth it to explore GC-related f-circRNAs and elucidate their mechanisms and clinical value in GC tumorigenesis. Fourth, most clinical samples for GC-related circRNA studies are obtained from advanced GC patients; thus, more samples from early GC and precancerous lesions are necessary to examine the early diagnostic value of circRNAs. Fifth, most studies on circRNA in GC were based on a relatively small number of samples; therefore, further validation on a larger cohort of patients (validation set) and the use of a clinic-related circRNAs database is needed. Sixth, exosomes as intercellular communicators can be secreted from tumor cells and often carry various signals such as noncoding RNAs, mRNAs, and proteins [99]. Therefore, introducing exosomal circRNAs as diagnostic biomarkers and therapeutics is promising. Finally, some circRNAs have been identified to regulate the chemoresistance of $\mathrm{GC}$, however, further studies are essential before they can be used clinically for GC treatment.

\section{Conflicts of Interest}

The authors declare that they have no conflict of interest.

\section{Acknowledgments}

This study was funded by the Second Batch of Research Projects in 2017 from Health and Family Planning Commission of Hunan Province (No. A2017014) and Hunan Provincial Innovation Foundation for Postgraduate (No. CX2018B645).

\section{References}

[1] F. Bray, J. Ferlay, I. Soerjomataram, R. L. Siegel, L. A. Torre, and A. Jemal, "Global cancer statistics 2018: GLOBOCAN estimates of incidence and mortality worldwide for 36 cancers in 185 countries," CA: a Cancer Journal for Clinicians, vol. 68, no. 6, pp. 394-424, 2018.

[2] A. P. Thrift and H. B. El-Serag, "Burden of Gastric Cancer," Clin Gastroenterol Hepatol, vol. 18, no. 3, pp. 534-542, 2020.

[3] R. M. Feng, Y. N. Zong, S. M. Cao, and R. H. Xu, "Current cancer situation in China: good or bad news from the 2018 Global Cancer Statistics?," Cancer Communications, vol. 39, no. $1,2019$.

[4] W. Chen, R. Zheng, P. D. Baade et al., "Cancer statistics in China, 2015," CA: A Cancer Journal for Clinicians, vol. 66, no. 2, pp. 115-132, 2016.

[5] J. K. Y. Hooi, W. Y. Lai, W. K. Ng et al., "Global Prevalence of Helicobacter pylori Infection: Systematic Review and Meta- 
Analysis," Gastroenterology, vol. 153, no. 2, pp. 420-429, 2017.

[6] Y. Hu, C. Huang, Y. Sun et al., "Morbidity and Mortality of Laparoscopic Versus Open D2 Distal Gastrectomy for Advanced Gastric Cancer: A Randomized Controlled Trial," Journal of Clinical Oncology, vol. 34, no. 12, pp. 1350-1357, 2016.

[7] R. Sitarz, M. Skierucha, J. Mielko, J. Offerhaus, R. Maciejewski, and W. Polkowski, "Gastric cancer: epidemiology, prevention, classification, and treatment," Cancer Management and Research, vol. Volume 10, pp. 239-248, 2018.

[8] X. Qi, Y. Liu, W. Wang et al., "Management of advanced gastric cancer: an overview of major findings from meta-analysis," Oncotarget, vol. 7, no. 47, pp. 78180-78205, 2016.

[9] H. Jin, P. S. Pinheiro, K. E. Callahan, and S. F. Altekruse, "Examining the gastric cancer survival gap between Asians and whites in the United States," Gastric Cancer, vol. 20, no. 4, pp. 573-582, 2017.

[10] M. A. Shah, "Targeted therapies in gastric cancer-the dawn of a new era," Nature Reviews. Clinical Oncology, vol. 11, no. 1, pp. 10-11, 2014.

[11] Y. Shao, J. Li, R. Lu et al., "Global circular RNA expression profile of human gastric cancer and its clinical significance," Cancer Medicine, vol. 6, no. 6, pp. 1173-1180, 2017.

[12] S. Memczak, M. Jens, A. Elefsinioti et al., "Circular RNAs are a large class of animal RNAs with regulatory potency," Nature, vol. 495, no. 7441, pp. 333-338, 2013.

[13] P. G. Zaphiropoulos, "Exon skipping and circular RNA formation in transcripts of the human cytochrome P-450 2C18 gene in epidermis and of the rat androgen binding protein gene in testis," Molecular and Cellular Biology, vol. 17, no. 6, pp. 2985-2993, 1997.

[14] S. Starke, I. Jost, O. Rossbach et al., "Exon circularization requires canonical splice signals," Cell Reports, vol. 10, no. 1, pp. 103-111, 2015.

[15] D. Liang, D. C. Tatomer, Z. Luo et al., "The Output of Protein-Coding Genes Shifts to Circular RNAs When the Pre-mRNA Processing Machinery Is Limiting," Molecular Cell, vol. 68, no. 5, pp. 940-954.e3, 2017.

[16] M. C. Kramer, D. Liang, D. C. Tatomer et al., "Combinatorial control of Drosophila circular RNA expression by intronic repeats, hnRNPs, and SR proteins," Genes \& Development, vol. 29, no. 20, pp. 2168-2182, 2015.

[17] S. J. Conn, K. A. Pillman, J. Toubia et al., “The RNA binding protein quaking regulates formation of circRNAs," Cell, vol. 160, no. 6, pp. 1125-1134, 2015.

[18] R. Ashwal-Fluss, M. Meyer, N. R. Pamudurti et al., "circRNA Biogenesis Competes with Pre-mRNA Splicing," Molecular Cell, vol. 56, no. 1, pp. 55-66, 2014.

[19] W. R. Jeck, J. A. Sorrentino, K. Wang et al., "Circular RNAs are abundant, conserved, and associated with ALU repeats," RNA, vol. 19, no. 2, pp. 141-157, 2013.

[20] X. O. Zhang, H. B. Wang, Y. Zhang, X. Lu, L. L. Chen, and L. Yang, "Complementary Sequence-Mediated Exon Circularization," Cell, vol. 159, no. 1, pp. 134-147, 2014.

[21] Y. Zhang, X. O. Zhang, T. Chen et al., "Circular Intronic Long Noncoding RNAs,” Molecular Cell, vol. 51, no. 6, pp. 792806, 2013.

[22] M. S. Mendoza-Figueroa, E. E. Alfonso-Maqueira, C. Vélez et al., "Postsplicing-Derived Full-Length Intron Circles in the Protozoan Parasite Entamoeba histolytica," Frontiers in Cellular and Infection Microbiology, vol. 8, no. 255, 2018.

[23] Z. Li, C. Huang, C. Bao et al., "Exon-intron circular RNAs regulate transcription in the nucleus," Nature Structural \& Molecular Biology, vol. 22, no. 3, pp. 256-264, 2015.

[24] Q. Hu and T. Zhou, "EIciRNA-mediated gene expression: tunability and bimodality,” FEBS Letters, vol. 592, no. 20, pp. 3460-3471, 2018.

[25] M. Englert, K. Sheppard, A. Aslanian, J. R. Yates, and D. Soll, "Archaeal 3'-phosphate RNA splicing ligase characterization identifies the missing component in tRNA maturation," Proceedings of the National Academy of Sciences, vol. 108, no. 4, pp. 1290-1295, 2011.

[26] C. A. Schmidt, J. J. Noto, G. S. Filonov, and A. G. Matera, "A Method for Expressing and Imaging Abundant, Stable, Circular RNAs In Vivo Using tRNA Splicing," Methods in Enzymology, vol. 572, pp. 215-236, 2016.

[27] Z. Lu, G. S. Filonov, J. J. Noto et al., "Metazoan tRNA introns generate stable circular RNAs in vivo," RNA, vol. 21, no. 9, pp. 1554-1565, 2015.

[28] T. B. Hansen, T. I. Jensen, B. H. Clausen et al., "Natural RNA circles function as efficient microRNA sponges," Nature, vol. 495, no. 7441, pp. 384-388, 2013.

[29] W. Yang, W. W. Du, X. Li, A. J. Yee, and B. B. Yang, "Foxo3 activity promoted by non-coding effects of circular RNA and Foxo3 pseudogene in the inhibition of tumor growth and angiogenesis," Oncogene, vol. 35, no. 30, pp. 3919-3931, 2016.

[30] M. Kapsimali, W. P. Kloosterman, E. de Bruijn, F. Rosa, R. H. A. Plasterk, and S. W. Wilson, "MicroRNAs show a wide diversity of expression profiles in the developing and mature central nervous system," Genome Biology, vol. 8, no. 8, 2007.

[31] M. Hu, S. Zhu, S. Xiong, X. Xue, and X. Zhou, "MicroRNAs and the PTEN/PI3K/Akt pathway in gastric cancer (Review)," Oncology Reports, vol. 41, no. 3, pp. 1439-1454, 2019.

[32] H. Pan, T. Li, Y. Jiang et al., "Overexpression of Circular RNA ciRS-7 Abrogates the Tumor Suppressive Effect of miR-7 on Gastric Cancer via PTEN/PI3K/AKT Signaling Pathway," Journal of Cellular Biochemistry, vol. 119, no. 1, pp. 440446, 2018.

[33] J. Quan, D. Dong, Y. Lun et al., "Circular RNA circHIAT1 inhibits proliferation and epithelial-mesenchymal transition of gastric cancer cell lines through downregulation of miR21," Journal of Biochemical and Molecular Toxicology, vol. 34, no. 4, article e22458, 2020.

[34] H. Song, Y. Xu, T. Xu et al., "CircPIP5K1A activates KRT80 and PI3K/AKT pathway to promote gastric cancer development through sponging miR-671-5p," Biomedicine \& Pharmacotherapy, vol. 126, article 109941, 2020.

[35] B. Sun, H. Sun, Q. Wang et al., "Circular RNA circMAN2B2 promotes growth and migration of gastric cancer cells by down-regulation of miR-145," Journal of Clinical Laboratory Analysis, no. article e23215, 2020.

[36] X. Zhang, S. Wang, H. Wang et al., "Circular RNA circNRIP1 acts as a microRNA-149-5p sponge to promote gastric cancer progression via the AKT1/mTOR pathway," Molecular Cancer, vol. 18, no. 1, 2019.

[37] Z. Wang, K. Ma, S. Pitts et al., "Novel circular RNA circNF1 acts as a molecular sponge, promoting gastric cancer by absorbing miR-16," Endocrine-Related Cancer, vol. 26, no. 3, pp. 265-277, 2019. 
[38] T. Liu, S. Liu, Y. Xu et al., "Circular RNA-ZFR Inhibited Cell Proliferation and Promoted Apoptosis in Gastric Cancer by Sponging miR- $_{130} \mathrm{a} / \mathrm{miR}-107$ and Modulating PTEN," Cancer Research and Treatment, vol. 50, no. 4, pp. 1396-1417, 2018.

[39] X. Dai, X. Guo, J. Liu et al., "Circular RNA circGRAMD1B inhibits gastric cancer progression by sponging miR-130a$3 \mathrm{p}$ and regulating PTEN and p21 expression," Aging, vol. 11, no. 21, pp. 9689-9708, 2019.

[40] S. Wang, X. Zhang, Z. Li et al., "Circular RNA profile identifies circOSBPL10 as an oncogenic factor and prognostic marker in gastric cancer," Oncogene, vol. 38, no. 44, pp. 69857001, 2019.

[41] J. Cheng, H. Zhuo, M. Xu et al., "Regulatory network of circRNA-miRNA-mRNA contributes to the histological classification and disease progression in gastric cancer," Journal of Translational Medicine, vol. 16, no. 1, 2018.

[42] X. Zhao, Q. Zhong, X. Cheng et al., "miR-449c-5p availability is antagonized by circ-NOTCH1 for MYC-induced NOTCH1 upregulation as well as tumor metastasis and stemness in gastric cancer," Journal of Cellular Biochemistry, 2020.

[43] J. Chen, Y. Li, Q. Zheng et al., "Circular RNA profile identifies circPVT1 as a proliferative factor and prognostic marker in gastric cancer," Cancer Letters, vol. 388, pp. 208-219, 2017.

[44] Y.-y. Liu, L.-y. Zhang, and W.-z. Du, "Circular RNA circ$\mathrm{PVT}_{1}$ contributes to paclitaxel resistance of gastric cancer cells through regulates $\mathrm{ZEB}_{1}$ expression by sponging miR124-3p,” Bioscience Reports, vol. 39, no. 12, 2019.

[45] Z. Zhu, Z. Rong, Z. Luo et al., "Circular RNA circNHSL1 promotes gastric cancer progression through the miR-13063p/SIX1/vimentin axis," Molecular Cancer, vol. 18, no. 1, 2019.

[46] X. Li, M. He, J. Guo, and T. Cao, "Upregulation of circular RNA circ-ERBB2 predicts unfavorable prognosis and facilitates the progression of gastric cancer via miR-503/CACUL1 and miR-637/MMP-19 signaling," Biochemical and Biophysical Research Communications, vol. 511, no. 4, pp. 926-930, 2019.

[47] T. Chohan, H. Qian, Y. Pan, and J. Chen, "Cyclin-Dependent Kinase-2 as a Target for Cancer Therapy: Progress in the Development of CDK2 Inhibitors as Anti-Cancer Agents," Current Medicinal Chemistry, vol. 22, no. 2, pp. 237-263, 2015.

[48] W. W. Du, W. Yang, E. Liu, Z. Yang, P. Dhaliwal, and B. B. Yang, "Foxo ${ }_{3}$ circular RNA retards cell cycle progression via forming ternary complexes with $\mathrm{p}_{21}$ and $\mathrm{CDK}_{2}$," Nucleic Acids Research, vol. 44, no. 6, pp. 2846-2858, 2016.

[49] I. Grammatikakis, K. Abdelmohsen, and M. Gorospe, "Posttranslational control of HuR function," Wiley Interdisciplinary Reviews: RNA, vol. 8, no. 1, article e1372, 2017.

[50] Y. Chen, F. Yang, E. Fang et al., "Circular RNA circAGO2 drives cancer progression through facilitating HuR-repressed functions of AGO2-miRNA complexes," Cell Death and Differentiation, vol. 26, no. 7, pp. 1346-1364, 2019.

[51] F. Yang, A. Hu, D. Li et al., "Circ-HuR suppresses HuR expression and gastric cancer progression by inhibiting CNBP transactivation," Molecular Cancer, vol. 18, no. 1, pp. 158-174, 2019.

[52] J. Fang, H. Hong, X. Xue et al., "A novel circular RNA, circFAT1(e2), inhibits gastric cancer progression by targeting miR-548g in the cytoplasm and interacting with YBX1 in the nucleus," Cancer Letters, vol. 442, no. 1, pp. 222-232, 2019.

[53] Y. Hong, H. Qin, Y. Li et al., "FNDC3B circular RNA promotes the migration and invasion of gastric cancer cells via the regulation of E-cadherin and CD44 expression," Journal of Cellular Physiology, vol. 234, no. 11, pp. 19895-19910, 2019.

[54] M. Jie, Y. Wu, M. Gao et al., "CircMRPS35 suppresses gastric cancer progression via recruiting KAT7 to govern histone modification," Molecular Cancer, vol. 19, no. 1, 2020.

[55] C. E. Aitken and J. R. Lorsch, "A mechanistic overview of translation initiation in eukaryotes," Nature Structural \& Molecular Biology, vol. 19, no. 6, pp. 568-576, 2012.

[56] S. Kong, M. Tao, X. Shen, and S. Ju, "Translatable circRNAs and lncRNAs: Driving mechanisms and functions of their translation products," Cancer Letters, vol. 483, pp. 59-65, 2020.

[57] I. Legnini, G. Di Timoteo, F. Rossi et al., "Circ-ZNF609 Is a Circular RNA that Can Be Translated and Functions in Myogenesis,” Molecular Cell, vol. 66, no. 1, pp. 22-37.e9, 2017.

[58] M. Zhang, N. Huang, X. Yang et al., "A novel protein encoded by the circular form of the SHPRH gene suppresses glioma tumorigenesis," Oncogene, vol. 37, no. 13, pp. 1805-1814, 2018.

[59] M. Zhang, K. Zhao, X. Xu et al., "A peptide encoded by circular form of _LINC-PINT_ suppresses oncogenic transcriptional elongation in glioblastoma," Nature Communications, vol. 9, no. 1, 2018.

[60] K. D. Meyer, D. P. Patil, J. Zhou et al., "5' UTR m6A Promotes Cap-Independent Translation,” Cell, vol. 163, no. 4, pp. 999-1010, 2015.

[61] Y. Yang, X. Fan, M. Mao et al., "Extensive translation of circular RNAs driven by $\mathrm{N}^{6}$-methyladenosine," Cell Research, vol. 27, no. 5, pp. 626-641, 2017.

[62] H. D. Sun, Z. P. Xu, Z. Q. Sun et al., "Down-regulation of circPVRL3 promotes the proliferation and migration of gastric cancer cells," Scientific Reports, vol. 8, no. 1, article 10111, 2018.

[63] F. Jiang, F. Hong, M. W. Shah, and X. Shen, "Circular RNAs as diagnostic biomarkers in gastric cancer: A meta-analysis review," Pathology - Research and Practice, vol. 215, no. 6, article 152419, p. 152419, 2019.

[64] K. W. Wang and M. Dong, "Role of circular RNAs in gastric cancer: recent advances and prospects," World Journal of Gastrointestinal Oncology, vol. 11, no. 6, pp. 459-469, 2019.

[65] P. Li, H. Chen, S. Chen et al., "Circular RNA 0000096 affects cell growth and migration in gastric cancer," British Journal of Cancer, vol. 116, no. 5, pp. 626-633, 2017.

[66] J. Lu, P. Y. Zhang, J. W. Xie et al., "Hsa_circ_0000467 promotes cancer progression and serves as a diagnostic and prognostic biomarker for gastric cancer," Journal of Clinical Laboratory Analysis, vol. 33, no. 3, article e22726, 2018.

[67] S. Chen, T. Li, Q. Zhao, B. Xiao, and J. Guo, "Using circular RNA hsa_circ_0000190 as a new biomarker in the diagnosis of gastric cancer," Clinica Chimica Acta, vol. 466, pp. 167171, 2017.

[68] S. Kong, Q. Yang, C. Tang, T. Wang, X. Shen, and S. Ju, "Identification of hsa_circ_0001821 as a novel diagnostic biomarker in gastric cancer via comprehensive circular RNA profiling," Frontiers in Genetics, vol. 10, 2019. 
[69] A. Ivanov, S. Memczak, E. Wyler et al., "Analysis of Intron Sequences Reveals Hallmarks of Circular RNA Biogenesis in Animals," Cell Reports, vol. 10, no. 2, pp. 170-177, 2015.

[70] W. Sui, Z. Shi, W. Xue et al., "Circular RNA and gene expression profiles in gastric cancer based on microarray chip technology," Oncology Reports, vol. 37, no. 3, pp. 1804-1814, 2017.

[71] D. Rong, C. Dong, K. Fu, H. Wang, W. Tang, and H. Cao, "Upregulation of circ_0066444 promotes the proliferation, invasion, and migration of gastric cancer cells," OncoTargets and Therapy, vol. Volume 11, pp. 2753-2761, 2018.

[72] J. Wei, W. Wei, H. Xu et al., "Circular RNA hsa_circRNA_ 102958 may serve as a diagnostic marker for gastric cancer," Cancer Biomarkers, vol. 27, no. 2, pp. 139-145, 2020.

[73] Q. Zhao, S. Chen, T. Li, B. Xiao, and X. Zhang, "Clinical values of circular RNA 0000181 in the screening of gastric cancer," Journal of Clinical Laboratory Analysis, vol. 32, no. 4, article e22333, 2018.

[74] H. Sun, W. Tang, D. Rong et al., "Hsa_circ_0000520, a potential new circular RNA biomarker, is involved in gastric carcinoma," Cancer Biomarkers, vol. 21, no. 2, pp. 299-306, 2018.

[75] T. Li, Y. Shao, L. Fu et al., "Plasma circular RNA profiling of patients with gastric cancer and their droplet digital RT-PCR detection," Journal of Molecular Medicine, vol. 96, no. 1, pp. 85-96, 2018.

[76] X. Tao, Y. Shao, R. Lu et al., "Clinical significance of hsa_circ_ 0000419 in gastric cancer screening and prognosis estimation," Pathology - Research and Practice, vol. 216, no. 1, article 152763, 2020.

[77] J. Lu, P.-y. Zhang, J.-w. Xie et al., "Circular RNA hsa_circ_ 0006848 Related to Ribosomal Protein L6 Acts as a Novel Biomarker for Early Gastric Cancer," Disease Markers, vol. 2019, Article ID 3863458, 13 pages, 2019.

[78] M. Huang, Y. R. He, L. C. Liang, Q. Huang, and Z. Q. Zhu, "Circular RNA hsa_circ_0000745 may serve as a diagnostic marker for gastric cancer," World Journal of Gastroenterology, vol. 23, no. 34, pp. 6330-6338, 2017.

[79] Y. Wang, S. Xu, Y. Chen, X. Zheng, T. Li, and J. Guo, "Identification of hsa_circ_0005654 as a new early biomarker of gastric cancer," Cancer Biomarkers, vol. 26, no. 4, pp. 403410, 2019.

[80] W.-h. Li, Y.-c. Song, H. Zhang et al., "Decreased Expression of Hsa_circ_00001649 in Gastric Cancer and Its Clinical Significance," Disease Markers, vol. 2017, Article ID 4587698, 6 pages, 2017.

[81] Y. Shao, L. Chen, R. Lu et al., "Decreased expression of hsa_ circ_0001895 in human gastric cancer and its clinical significances," Tumor Biology, vol. 39, no. 4, 2017.

[82] M. Tian, R. Chen, T. Li, and B. Xiao, "Reduced expression of circRNA hsa_circ_0003159 in gastric cancer and its clinical significance," Journal of Clinical Laboratory Analysis, vol. 32, no. 3, article e22281, 2018.

[83] R. Lu, Y. Shao, X. Tao, G. Ye, B. Xiao, and J. Guo, "Clinical significances of hsa_circ_0067582 and hsa_circ_0005758 in gastric cancer tissues," Journal of Clinical Laboratory Analysis, vol. 33, no. 9, article e22984, 2019.

[84] P. Li, S. Chen, H. Chen et al., "Using circular RNA as a novel type of biomarker in the screening of gastric cancer," Clinica Chimica Acta, vol. 444, pp. 132-136, 2015.
[85] R. Lu, Y. Shao, G. Ye, B. Xiao, and J. Guo, "Low expression of hsa_circ_0006633 in human gastric cancer and its clinical significances," Tumor Biology, vol. 39, no. 6, 2017.

[86] W. Tang, K. Fu, H. Sun, D. Rong, H. Wang, and H. Cao, "CircRNA microarray profiling identifies a novel circulating biomarker for detection of gastric cancer," Molecular Cancer, vol. 17, no. 1, 2018.

[87] Y. K. Peng, K. Pu, H. X. Su et al., "Circular RNA hsa_circ_ ${ }_{0010} 88_{2}$ promotes the progression of gastric cancer via regulation of the $\mathrm{PI}_{3} \mathrm{~K} / \mathrm{Akt} / \mathrm{mTOR}$ signaling pathway," European Review for Medical and Pharmacological Sciences, vol. 24, no. 3, pp. 1142-1151, 2020.

[88] L. Zhang, X. Chang, T. Zhai et al., "A novel circular RNA, circ-ATAD1, contributes to gastric cancer cell progression by targeting miR-140-3p/YY1/PCIF1 signaling axis," Biochemical and Biophysical Research Communications, vol. 525, no. 4, pp. 841-849, 2020.

[89] L. Wu, D. Liu, and Y. Yang, "Enhanced expression of circular RNA circ-DCAF6 predicts adverse prognosis and promotes cell progression via sponging miR-1231 and miR-1256 in gastric cancer," Experimental and Molecular Pathology, vol. 110, article 104273, p. 104273, 2019.

[90] J. Zhang, H. Liu, L. Hou et al., "Circular RNA_LARP4 inhibits cell proliferation and invasion of gastric cancer by sponging miR-424-5p and regulating LATS1 expression," Molecular Cancer, vol. 16, no. 1, 2017.

[91] J. He, J. Chen, B. Ma, L. Jiang, and G. Zhao, "CircLMTK 2 acts as a novel tumor suppressor in gastric cancer," Bioscience Reports, vol. 39, no. 5, 2019.

[92] Y. Zhang, J. Li, J. Yu et al., "Circular RNAs signature predicts the early recurrence of stage III gastric cancer after radical surgery," Oncotarget, vol. 8, no. 14, pp. 2293622943, 2017.

[93] H. Arai and T. E. Nakajima, "Recent developments of systemic chemotherapy for gastric cancer," Cancers, vol. 12, no. 5, 2020.

[94] C. Cui, J. Yang, X. Li, D. Liu, L. Fu, and X. Wang, "Functions and mechanisms of circular RNAs in cancer radiotherapy and chemotherapy resistance," Molecular Cancer, vol. 19, no. $1,2020$.

[95] X. Huang, Z. Li, Q. Zhang et al., "Circular RNA AKT3 upregulates PIK3R1 to enhance cisplatin resistance in gastric cancer via miR-198 suppression," Molecular Cancer, vol. 18, no. $1,2019$.

[96] X. X. Huang, Q. Zhang, H. Hu et al., “A novel circular RNA circFN1 enhances cisplatin resistance in gastric cancer via sponging miR-182-5p," Journal of Cellular Biochemistry, 2020.

[97] Q. Wu, H. Wang, L. Liu, K. Zhu, W. Yu, and J. Guo, "Hsa_ circ_0001546 acts as a miRNA-421 sponge to inhibit the chemoresistance of gastric cancer cells via ATM/Chk2/p53dependent pathway," Biochemical and Biophysical Research Communications, vol. 521, no. 2, pp. 303-309, 2020.

[98] L. Ma, Z. Wang, M. Xie et al., "Silencing of_circRACGAP1_ sensitizes gastric cancer cells to apatinib via modulating autophagy by targeting _miR-3657_ and _ATG7_, Cell Death \& Disease, vol. 11, no. 3, 2020.

[99] Y. Yan, G. Fu, Y. Ye, and L. Ming, "Exosomes participate in the carcinogenesis and the malignant behavior of gastric cancer," Scandinavian Journal of Gastroenterology, vol. 52, no. 5, pp. 499-504, 2017. 
[100] J. Lu, Y.-h. Wang, C. Yoon et al., "Circular RNA circRanGAP1 regulates VEGFA expression by targeting miR877-3p to facilitate gastric cancer invasion and metastasis," Cancer Letters, vol. 471, pp. 38-48, 2020.

[101] X. Li, L. Yang, and L. L. Chen, "The biogenesis, functions, and challenges of circular RNAs," Molecular Cell, vol. 71, no. 3, pp. 428-442, 2018.

[102] J. Guarnerio, M. Bezzi, J. C. Jeong et al., "Oncogenic role of fusion-circRNAs derived from cancer-associated chromosomal translocations," Cell, vol. 165, no. 2, pp. 289302, 2016.

[103] K. Wu, X. Liao, Y. Gong et al., "Circular RNA F-circSR derived from SLC34A2-ROS1 fusion gene promotes cell migration in non-small cell lung cancer," Molecular Cancer, vol. 18, no. 1, 2019.

[104] S. Tan, Q. Gou, W. Pu et al., "Circular RNA F-circEA produced from EML4-ALK fusion gene as a novel liquid biopsy biomarker for non-small cell lung cancer," Cell Research, vol. 28, no. 6, pp. 693-695, 2018.

[105] Y. He, Y. Wang, L. Liu et al., "Circular RNA circ_0006282 Contributes to the Progression of Gastric Cancer by Sponging miR-155 to Upregulate the Expression of FBXO22," OncoTargets and Therapy, vol. Volume 13, pp. 1001-1010, 2020.

[106] S. Lin, S. Song, R. Sun et al., "Oncogenic circular RNA Hsacirc-000684 interacts with microRNA-186 to upregulate ZEB1 in gastric cancer," The FASEB Journal, vol. 34, no. 6, 2020.

[107] F. Shen, P. Liu, Z. Xu et al., "CircRNA_001569 promotes cell proliferation through absorbing miR-145 in gastric cancer," The Journal of Biochemistry, vol. 165, no. 1, pp. 27-36, 2019.

[108] R. Li, B. Wu, J. Xia, L. Ye, and X. Yang, "Circular RNA hsa_ circRNA_102958 promotes tumorigenesis of colorectal cancer via miR-585/CDC25B axis," Cancer Management and Research, vol. Volume 11, pp. 6887-6893, 2019.

[109] W. Wei, X. Mo, L. Yan et al., "Circular RNA profiling reveals that circRNA_104433 regulates cell growth by targeting miR497-5p in gastric cancer," Cancer Management and Research, vol. Volume 12, pp. 15-30, 2020.

[110] C. Cao, S. Han, Y. Yuan et al., "Downregulated Circular RNA hsa_circ_0000291 Suppresses Migration And Proliferation Of Gastric Cancer Via Targeting The miR-183/ITGB1 Axis," Cancer Management and Research, vol. Volume 11, pp. 9675-9683, 2019.

[111] B. Li, M. Jin, F. Cao et al., "Hsa_circ_0017639 expression promotes gastric cancer proliferation and metastasis by sponging miR-224-5p and upregulating USP3," Gene, vol. 750, article 144753, 2020.

[112] Z. Chen, H. Ju, T. Zhao et al., "hsa_circ_0092306 targeting miR-197-3p promotes gastric cancer development by regulating PRKCB in MKN-45 cells," Molecular Therapy - Nucleic Acids, vol. 18, pp. 617-626, 2019.

[113] Z. Zhang, H. Wu, Z. Chen, G. Li, and B. Liu, "Circular RNA $\mathrm{ATXN}_{7}$ promotes the development of gastric cancer through sponging miR- ${ }_{4319}$ and regulating $\mathrm{ENTPD}_{4}$," Cancer Cell International, vol. 20, no. 1, 2020.

[114] H. Liu, Y. Liu, Z. Bian et al., "Circular RNA YAP1 inhibits the proliferation and invasion of gastric cancer cells by regulating the miR-367-5p/p27 Kip1 axis," Molecular Cancer, vol. 17, no. $1,2018$.
[115] G. Deng, T. Mou, J. He et al., "Circular RNA circRHOBTB3 acts as a sponge for miR-654-3p inhibiting gastric cancer growth," Journal of Experimental \& Clinical Cancer Research, vol. 39, no. 1, 2020.

[116] Z. Luo, Z. Rong, J. Zhang et al., "Circular RNA circCCDC9 acts as a miR-6792-3p sponge to suppress the progression of gastric cancer through regulating CAV1 expression," Molecular Cancer, vol. 19, no. 1, 2020.

[117] J. Lu, P. Y. Zhang, P. Li et al., "Circular RNA hsa_circ_0001368 suppresses the progression of gastric cancer by regulating miR-6506-5p/FOXO3 axis," Biochemical and Biophysical Research Communications, vol. 512, no. 1, pp. 29-33, 2019. 